\title{
A Novel Version of HPM Coupled with the PSEM Method for Solving the Blasius Problem
}

\author{
Uriel Filobello-Nino, ${ }^{1}$ Hector Vazquez-Leal $\mathbb{D}^{1,}{ }^{1,2}$ Jesus Huerta-Chua, ${ }^{3}$ \\ Victor Manuel Jimenez-Fernandez, ${ }^{1}$ Mario Alberto Sandoval-Hernandez, \\ Enrique Delgado-Alvarado, ${ }^{3}$ and Victor Manuel Tlapa-Carrera $\mathbb{D}^{3}$ \\ ${ }^{1}$ Facultad de Instrumentación Electrónica, Universidad Veracruzana, Cto. Gonzalo Aguirre Beltrán S/N, Xalapa 91000, \\ Veracruz, Mexico \\ ${ }^{2}$ Consejo Veracruzano de Investigación Científica y Desarrollo Tecnológico (COVEICYDET), Av Rafael Murillo Vidal No. 1735, \\ Cuauhtémoc, Xalapa 91069, Veracruz, Mexico \\ ${ }^{3}$ Instituto Tecnológico Superior de Poza Rica, Tecnológico Nacional de México, \\ Luis Donaldo Colosio Murrieta S/N, Arroyo Del Maíz, C.P., Poza Rica 93230, Veracruz, Mexico \\ ${ }^{4}$ CBTis 190 DGETI, Av 15, Venustiano Carranza, Carranza 2da Sección, Boca Del Río 94297, Veracruz, Mexico
}

Correspondence should be addressed to Hector Vazquez-Leal; hvazquez@uv.mx

Received 20 May 2021; Revised 2 July 2021; Accepted 20 July 2021; Published 6 August 2021

Academic Editor: Marcio Eisencraft

Copyright (c) 2021 Uriel Filobello-Nino et al. This is an open access article distributed under the Creative Commons Attribution License, which permits unrestricted use, distribution, and reproduction in any medium, provided the original work is properly cited.

\begin{abstract}
This work studies the nonlinear differential equation that models the Blasius problem (BP) which is of great importance in fluid dynamics. The aim is to obtain an approximate analytical expression that adequately describes the phenomenon considered. To find such approximation, we propose a new method denominated powered homotopy perturbation (PHPM). Unlike HPM algorithm, the successive integration process generated by PHPM will consider zero the constants of integration in each approximation, except the last one. In the same way, PHPM will propose an adequate initial trial function provided of some unknown parameters in such a way that it will not evaluate the initial conditions in the iterations of the process; therefore, this set of parameters will be employed with the purpose of adjusting in the best accurate way the proposed approximation at the final part of the process. As a matter of fact, we will note from this analysis that the proposed solution is compact and easy to evaluate and involves a sum of five exponential functions plus a linear part of two terms, which is ideal for practical applications. With the purpose to get a better approximation, we find useful to combine PHPM with the power series extender method (PSEM) which implies to add to the PHPM solution one rational function with parameters to adjust. From this proposal, we find an approximate solution competitive with others from the literature.
\end{abstract}

\section{Introduction}

As it is well known, the fluid motion is divided into two clearly defined regions; the first one concerns the region near the object where the friction effect is appreciable and is known as boundary layer. On the other hand, the second region is that where these effects can be neglected $[1,2]$. The boundary layer is characterized for being the region where the fluid velocity parallel to the surface is less $99 \%$ of the free stream velocity. Nevertheless, even in the case of a laminar flow, the solution of the equations describing the laminar boundary layer is complicated [1,2]. This work will search for a solution for the BP, which describes the two-dimensional steady state viscous flow over a semi-infinite flat plate. Specifically, the aim is to get an analytical approximate solution that adequately describes this relevant fluid mechanics problem. The difficulties of these scientific problems give rise to the proposal of new methods with the end to obtain solutions to the differential equations that govern nonlinear problems such as the one proposed here; however, 
the search for such solutions is not an easy task and justifies all the research efforts carried out on this topic. In effect, an exact solution to the proposed nonlinear problem can rarely be obtained [3], and for the same reason, several methods have been added to the best known classical methods. Next, we provide a list with some of most employed analytical methods in accordance with the literature: variational approaches [4-6], the tanh method [7], exp-function [8,9], Adomian's decomposition method [10-15], parameter expansion [16], the homotopy perturbation method (HPM) $[1,6,17-33]$, the perturbation method [34-36], the modified Taylor series method [37], the Picard method [38], the PSEM [39-42], the homotopy analysis method [25, 43], the variational iteration method [44], and the differential transform method [45], among others. Numerical methods play an important role with the purpose to provide algorithms to get approximations of differential equations [46-49]. This work introduces two novelties; on one side, it presents an effective modification of the standard HPM, the power homotopy perturbation method (PHPM), with the purpose of finding an analytical approximate solution for the BP. As it is well known, the HPM has been one of the most efficient methods proposed to get analytical approximate solutions for nonlinear problems. The HPM was first proposed by a Chinese mathematician Ji-Huan He to investigate a wide variety of nonlinear problems which arise in science and engineering. The HPM successfully couples the homotopy theory with the perturbation theory (but it is not restricted to small parameters as with traditional perturbation methods). The main idea is that a complicated problem is continuously deformed into a simple problem which is easy to solve in order to get an analytic exact or approximate solution [20]. On the other hand, we will solve the same problem, but now, the PHPM will provide a solution just for a part of the whole interval of the BP. Instead of looking for a solution for the other part of the interval and introducing a piece-wise kind solution [41] for the whole interval and even proposing, for instance, to express the final result in a compact expression by using the unit step function $U(x-a)$ [50], we will employ the PSEM to obtain an approximate solution valid for all the definition domains of the proposed problem. As a matter of fact, after comparing the approximate solution obtained for this work with other approximations from the literature, we will see that the combination of the PHPM and PSEM offers, indeed, potential for the approximate solution of nonlinear problems. The rest of this work is as follows: Section 2 introduces the basic idea of the standard HPM. Section 3 explains thePHPM. Additionally, Section 4 explains the basic concept of the PSEM. On the other hand, Section 5 presents, with some details, the necessary elements for the nonlinear problem to solve. Besides, Section 6 presents the application of the proposed methods, in the search for an approximate solution for the third-order nonlinear ordinary differential equation which describes the BP. Section 7 offers a whole discussion about the proposed solutions and their comparison with other approximations found in the literature. Finally, a brief conclusion of the relevant aspects of this work is given in Section 8 .

\section{Standard HPM}

The standard HPM was conceived with the end to approach various kinds of nonlinear problems $[19,20]$. The HPM is a combination of the classical perturbation technique and the homotopy (whose origin lies in the branch of mathematics denominated topology), but it is not restricted to small parameters as with traditional perturbation methods (to say PM). For example, the HPM requires neither small parameter nor linearization and usually requires few iterations to obtain solutions with good precision $[19,20]$.

To conceive how the HPM works, we consider a general nonlinear differential equation, which can be expressed as follows:

$$
A(u)-f(r)=0, \quad r \in \Omega,
$$

with the following boundary conditions:

$$
B\left(u, \frac{\partial u}{\partial n}\right)=0, \quad r \in \Gamma,
$$

where $A(u)$ is a general differential operator, $B$ is a boundary operator, $f(r)$ is a given analytical function, and $\Gamma$ is the domain boundary for $\Omega$. Next, assuming that $A$ can be divided into two operators $L$ and $N$, where $L$ is linear and $N$ nonlinear; (1) can be expressed as

$$
L(u)+N(u)-f(r)=0
$$

An homotopy can be constructed in accordance with $[19,20]$.

$$
H(U, p)=(1-p)\left[L(U)-L\left(u_{0}\right)\right]+p[L(U)+N(U)-f(r)]=0, \quad p \in[0,1], r \in \Omega
$$

or

$$
H(U, p)=L(U)-L\left(u_{0}\right)+p\left[L\left(u_{0}\right)+N(U)-f(r)\right]=0, \quad p \in[0,1], r \in \Omega,
$$


where $p$ is a homotopy parameter, whose values belong to the range of $[0,1] ; u_{0}$ is the first approximation for the solution of (3) that satisfies the boundary conditions.

Next, we will assume that the solution for (4) or (5) can be written as a power series of $p$ in the form

$$
U=v_{0}+v_{1} p+v_{2} p^{2}+\cdots,
$$

and after substituting (6) into (5) and equating identical powers of $p$ terms, there can be found values for the sequence $v_{o}, v_{1}, v_{2}, \ldots$ after solving a coupled system of differential equations.

$$
\begin{aligned}
L(U) & =L\left(v_{0}\right), \\
v_{0}(A) & =a, \\
v_{0}^{\prime}(A) & =b, \\
v_{0}^{\prime \prime}(A) & =c, \ldots, \\
L\left(v_{1}\right)+L\left(v_{0}\right)+N\left(v_{0}\right)-f(r) & =0, \\
v_{1}(A) & =0, \\
v_{1}^{\prime}(A) & =0, \\
v_{1}^{\prime \prime}(A) & =0, \ldots, \\
N\left(v_{0}, v_{1}\right) & =0, \\
v_{2}(A) & =0, \\
v_{2}^{\prime}(A) & =0, \\
v_{2}^{\prime \prime}(A) & =0, \ldots, \\
\ldots\left(v_{2}\right)+ & \\
L\left(v_{j}\right)+N\left(v_{0}, v_{1}, v_{2}, \ldots, v_{j-2}, v_{j-1}\right) & =0, \\
v_{j}(A) & =0, \\
v_{j}^{\prime}(A) & =0, \\
v_{j}^{\prime}(A) & =0, \ldots \\
\ldots &
\end{aligned}
$$

Taking the limit $p \longrightarrow 1$, an approximate solution for (1) is obtained as follows:

$$
U(x)=v_{0}(x)+v_{1}(x)+v_{2}(x)+v_{3}(x), \ldots,
$$

where $A$ denotes the starting point for the initial conditions and $a, b, c, \ldots$ are the initial conditions of the problem.

\section{The Proposed Method: Powered Homotopy Perturbation Method (PHPM)}

From the above, we note that the standard HPM establishes that the values of higher-order approximations $v_{1}, v_{2}, v_{3} \ldots$ have to be zero if they are evaluated in the initial condition value $x=A$. The proposed method PHPM essentially follows the same steps of the HPM, but unlike it, the successive integration process of the coupled system (7)-(10) is performed without evaluating the initial conditions mentioned by the HPM. In a sequence, the process of successive approximations (7)-(10) will be affected considering zero the constants of integration in each approximation, excepting the last one, which will keep all their integration constants that are originated from the process of solution. In this stage, one approximate solution for the problem to solve is obtained again considering the limit (11). Nevertheless, we note that this solution possesses some integration constants to evaluate, even other parameters that could rise of the freedom of the homotopy technique [22,23]. These parameters are evaluated from the initial conditions applied to (11) and the knowledge of other mathematical and physical properties of the nonlinear problem. Therefore, the solution for a problem is expressed in terms of solving an algebraic system of equations.

The scheme for the PHPM, analogous to (7)-(10), is

$$
\begin{aligned}
L(U) & =L\left(v_{0}\right), \\
L\left(v_{1}\right)+L\left(v_{0}\right)+N\left(v_{0}\right)-f(r) & =0, \\
L\left(v_{2}\right)+N\left(v_{0}, v_{1}\right) & =0 \\
& \ldots, \\
L\left(v_{j}\right)+N\left(v_{0}, v_{1}, v_{2}, \ldots, v_{j-2}, v_{j-1}\right) & =0,
\end{aligned}
$$

assuming that $j$ is the last iteration considered in order to provide an approximate solution for (1), and then, it is possible to rewrite (15) symbolically as

$$
v_{j}=L^{-1}\left(-N\left(v_{0}, v_{1}, v_{2}, \ldots, v_{j-2}, v_{j-1}\right)\right)+c_{1}+c_{2} x+c_{3}\left(\frac{x^{2}}{2}\right)+\cdots,
$$

where $L$ and $L^{-1}$ are inverse operators and $c 1, c 2, c 3, \ldots$ are constants to determine.

Taking the limit $p \longrightarrow 1$, it is possible to obtain an approximate solution for (1) as follows:

$$
U(x)=v_{0}(x)+v_{1}(x)+v_{2}(x)+v_{3}(x) \cdots+v_{j}(x) .
$$

The constants $c 1, c 2, c 3, \ldots$ are found from the conditions

$$
\begin{aligned}
U(A) & =a, \\
U^{\prime}(A) & =b, \\
U^{\prime \prime}(A) & =c, \ldots
\end{aligned}
$$

We will see that the Blasius problem is a good candidate to apply this scheme.

\section{Basic Concept of the PSEM}

In accordance with [39], we begin assuming a nonlinear differential equation which is expressed as follows:

$$
L(u)+N(u)-f(x)=0, \quad x \in \Omega .
$$

The boundary conditions of (19) can be expressed as 


$$
B\left(u, \frac{\partial u}{\partial n}\right)=0, \quad r \in \Gamma
$$

where $B$ is a boundary operator; $f(x)$ is a given analytical function; $\Gamma$ is the boundary for domain $\Omega$; and $L$ and $N$ represent linear and nonlinear operators, respectively. Finally, $\partial u / \partial n$ expresses differentiation along the normal drawn outwards from $\Omega$. The following step consists in assuming that the solution of (19) can be expressed as a power series:

$$
u=\sum_{k=0}^{\infty} c_{k} x^{k}
$$

where $c_{k}^{\prime} s$ represents the coefficients of the power series.

In accordance with PSEM flexibility, series (21) can be obtained by some approximate methods from the literature: the Taylor series method [37], power series method [39], homotopy perturbation method [1,17-23], perturbation method [34-36], homotopy analysis method [43], variational iteration method [44], differential transform method [45], and Adomian decomposition method [10-15], among others.

Next, we assume that the solution for differential equation (19) can be expressed as a finite sum of certain functions in accordance with [39].

$$
u=u_{0}+\sum_{i=0}^{n} f_{i}\left(x, u_{i}\right)
$$

or

$$
u=\frac{u_{0}+\sum_{i=0}^{n} f_{i}\left(x, u_{i}\right)}{1+\sum_{j=n+1}^{2 n} f_{j}\left(x, u_{j}\right)},
$$

where $u=u_{i}$ are, in principle, unknown constants to be determined by the PSEM, $f_{i}\left(x, u_{i}\right)$ are arbitrary trial functions, and $n$ and $2 n$ represent the orders of approximation of (22) and (23), respectively. As a matter of fact, from now on, we will designate (22) and (23) as the PSEM trial function (TF).

The next step consists in obtaining the Taylor series of (22) or (23) so that we get the power series of the form

$$
\begin{aligned}
& u=u_{0}+\sum_{i=0}^{n} P_{i, 0}+\sum_{i=0}^{n} \sum_{k=0}^{\infty} P_{i, k} x^{k}, \\
& u=u_{0}+\sum_{i=0}^{n} P_{i, 0}+\sum_{i=0}^{2 n} \sum_{k=0}^{\infty} P_{i, k} x^{k},
\end{aligned}
$$

where Taylor coefficients $P_{K}$ are expressed in terms of parameters $u_{i}$. In particular, $P_{i, 0}$ are the coefficients of the order zero Taylor expansion applied to the finite sum of functions (22) and rational function (23), respectively. Finally, we equate the coefficients of the corresponding power series (24) or (25) with (21) in order to get the values $u_{i}$. After substituting them into (22) or (23), we finally obtain the PSEM approximation.

\section{Problem Formulation}

This section will provide the sufficient elements required for the rest of this work. As an application of the PHPM, we will get an analytical approximate solution for the nonlinear third-order Blasius ordinary differential equation (BODE) which can be written in the following terms:

$$
\begin{aligned}
y^{\prime \prime \prime}(x)+\frac{1}{2} y(x) y^{\prime \prime}(x) & =0, \quad x \geq 0, \\
y(0) & =0 \\
y^{\prime}(0) & =0, \\
y^{\prime}(\infty) & =1,
\end{aligned}
$$

where $y(x)$ denotes the dimensionless stream function and $x$ is a dimensionless coordinate proportional to the perpendicular distance from the front of the plate, inversely proportional to the thickness of the boundary layer. As it is well known, the boundary layer is defined as the adjacent region to a solid surface where viscous forces are relevant and its thickness is defined where the derivative is $y^{\prime}=0.99$. From [51], it results that the values of $x$ and $y$ defining the boundary layer are related by $y(5)=3.28329$ (see Section 6).

On the other hand, Blasius found a series solution for system (26) and (27) [52] which is given by

$$
\begin{aligned}
y(x) & =\sum_{k=0}^{\infty}\left(\frac{-1}{2}\right)^{k} \frac{A_{k}\left(y^{\prime \prime}(0)\right)^{k+1}}{(3 k+2) !} x^{3 k+2}, \\
A_{0} & =A_{1}=1, \\
A_{k} & =\sum_{s=0}^{k-1}\left(\begin{array}{c}
3 k-1 \\
3 r
\end{array}\right) A_{s} A_{k-r-1}, \quad k \geq 2,
\end{aligned}
$$

and $y^{\prime \prime}(0)$ was numerically determined with the value $0.332057336[53,54]$.

Such as it occurs for every mathematical series, (28) converges only for a finite interval $[0, \phi]$ (see [51]), where $\phi$ is a number such that $\phi \approx 1.8894 / y^{\prime \prime}(0)$ or $\phi \approx 5.69$; therefore, this series does not totally describe the Blasius problem, given that it requires a solution for the whole interval $x \geq 0$.

Since the aim of this work is to provide an analytical approximate solution valid for the entire domain $[0, \infty)$ and not only for small $x$, it will result useful to express condition (27) $y^{\prime}(\infty)=1$ in the asymptotic form:

$$
y(x)=x+c, \quad x \longrightarrow \infty,
$$

for some constant value of $c$. As a matter of fact, we will see that the geometric determination of this parameter will be relevant for the solution of the asymptotic part of this problem. 


\section{Application of the PHPM with the End to Obtain Two Approximate Solutions for the Nonlinear Blasius Problem}

In accordance with the previous section, we have to find an approximate solution for BODE.

$$
y^{\prime \prime \prime}(x)+\frac{1}{2} y(x) y^{\prime \prime}(x)=0, \quad x \geq 0
$$

From Section 3, we establish the following homotopy, by using the flexibility of PHPM:

$$
(1-p)\left[y^{\prime \prime \prime}(x)\right]+p\left[y^{\prime \prime \prime}(x)+\frac{1}{2} y(x) y^{\prime \prime}(x)\right]=0
$$

or

$$
y^{\prime \prime \prime}(x)+\frac{p}{2} y(x) y^{\prime \prime}(x)=0 .
$$

Following PHPM algorithm, we will assume that (33) admits a series solution of the form

$$
y(x)=y_{0}(x)+y_{1}(x) p+y_{2}(x) p^{2}+y_{3}(x) p^{3}, \ldots,
$$

in such a way that, after substituting (34) into (33), we get the following system of linear differential equations:

$$
\begin{aligned}
& y_{1}^{\prime \prime}(x)=-\frac{1}{2} y_{0}(x) y_{0}^{\prime \prime}(x) \\
& y_{2}^{\prime \prime}(x)=-\frac{1}{2} y_{1}(x) y_{0}^{\prime \prime}(x)-\frac{1}{2} y_{0}(x) y_{1}^{\prime \prime}(x) \\
& y_{3}^{\prime \prime}(x)=-\frac{1}{2} y_{2}(x) y_{0}^{\prime \prime}(x)-\frac{1}{2} y_{0}(x) y_{2}^{\prime \prime}(x)-\frac{1}{2} y_{1}(x) y_{1}^{\prime}(x)
\end{aligned}
$$

and so on.

Next, we propose a solution depending of two parameters, from the asymptotic characteristics of the Blasius problem, and let it be

$$
y_{0}(x)=A e^{-B x}, \quad A, B>0,
$$

where positive quantities $A$ and $B$ will be determined from the properties of the problem.

Assuming that we keep until the third iteration of (34), we will solve (35)-(38).

Thus, by substituting (38) into (35), we get

$$
y_{1}^{\prime \prime}(x)=-\frac{1}{2} A^{2} B^{2} e^{-2 B x}
$$

and after integrating three times, without keeping the integration constants, we obtain

$$
y_{1}(x)=\frac{A^{2} e^{-2 B x}}{16 B} .
$$

In the same way, substituting (38) and (40) into (36), we get

$$
y_{2}^{\prime \prime}(x)=\frac{-5 A^{3} B e^{-3 B x}}{32} .
$$

Integrating three times, without keeping the integration constants, we obtain

$$
y_{2}(x)=\frac{5 A^{3} e^{-3 B x}}{864 B^{2}} .
$$

The third iteration results from replacing (38), (40), and (42) into (37) so that we get

$$
y_{3}^{\prime \prime}(x)=\frac{-50 A^{4} e^{-4 B x}}{1728}+\frac{A^{4} e^{-4 B x}}{256 B} .
$$

It is possible to integrate (43) in the same way three times; assuming that it is the last iteration, we have to add tree integration constants produced by tree integrations in such a way that we write

$$
y_{3}(x)=\frac{50 A^{4} e^{-4 B x}}{110592 B^{3}}-\frac{A^{4} e^{-4 B x}}{16384 B^{4}}+k_{1}+k_{2} x+\frac{k_{3}}{2} x^{2} \text {. }
$$

We note that, from asymptotic condition (30), it is required that the last term of (44) has to be zero; besides, from the same condition, it is clear that $k_{2}=1$. Therefore, (44) has to adopt the form

$$
y_{3}(x)=\frac{50 A^{4} e^{-4 B x}}{110592 B^{3}}-\frac{A^{4} e^{-4 B x}}{16384 B^{4}}+k_{1}+x .
$$

After taking the limit $p \longrightarrow 1$, we obtain an approximate solution for (26) with boundary conditions (27) as follows:

$$
y(x)=\sum_{k=0}^{3} y_{k}(x)
$$

see (38), (40), (42), and (45).

From the above, it is clear that (46) is an analytical approximate solution for (31) which satisfies the correct asymptotic condition (30).

In accordance with (46), we have obtained an approximate solution with the schematic form

$$
y(x)=Y(x)+x+k_{1}
$$

where $Y(x)$ represents all the terms of (46) which contain only negative exponentials.

As it was already mentioned, the boundary layer is defined as the adjacent region to a solid surface where viscous forces are relevant and its thickness is defined where the derivative is $y^{\prime}=0.99$.

Also, it was mentioned that this point corresponds to the coordinates $y(5)=3.28329$, so that, for $x=5$, it is expected that (47) adopts the asymptotic form (30) with good precision. Thus, $Y(x) \approx 0$ and

$$
y(x) \cong x+k_{1} .
$$

Geometrically, (48) is the equation of the tangent line to the point $(5,3.28329)$ and $k_{1}$ can be determined by substituting the coordinates into (48) to obtain the equation 


$$
y(x)=x-1.7168
$$

Thus, (47) can be written as

$$
y(x)=Y(x)+x-1.7168
$$

which ensures the asymptotic form of the solution, even from the point for which $y^{\prime}=0.99$.

With the purpose of ensuring that (50) satisfies the initial conditions of the problem, it is necessary to adequately evaluate the values of the constants $A$ and $B$. For it, we will substitute the first condition and the second condition of (27) into (50) in order to get an algebraic system for the unknown quantities.

$$
\begin{aligned}
Y(0)-1.7168 & =0 \\
Y^{\prime}(0)+1 & =0
\end{aligned}
$$

The solution for nonlinear system (51) is given by the values

$$
\begin{aligned}
& A=1.3797, \\
& B=0.46578 .
\end{aligned}
$$

Thus, substituting these values into (50), we get an approximate solution for (26) with boundary conditions (27).

$$
y(x)=0.1660285 x^{2}-0.0004594243 x^{5}+2.4971813 \times 10^{-6} x^{8}-1.4276972 \times 10^{-8} x^{11}+\cdots
$$

$$
\begin{aligned}
& A=14.29862177, \\
& B=1.613153514 .
\end{aligned}
$$

Therefore, by substituting (54) into (50), we get an analytical approximate solution for the Blasius problem valid for $[3, \infty)$.

On the other hand, in order to get a solution for $(0,3]$, we will employ the PSEM. As explained in Section 4, next we assume the following rational function valid for the abovementioned interval: (45).

After solving the resulting $2 \times 2$ nonlinear system, we get

$$
y_{2}(x)=\frac{\left(a_{2} x^{2}+a_{1} x+a_{0}\right) e^{-2 x}}{\left(a_{9} x^{9}+a_{8} x^{8}+a_{7} x^{7}+a_{6} x^{6}+a_{5} x^{5}+a_{4} x^{4}+a_{3} x^{3}+a_{2} x^{2}+a_{1} x+1\right)} .
$$

The coefficients of (55) $a^{\prime} s$ and $b^{\prime} s$ are determined by using PSEM algorithm, and the presence of $\exp (-2 x)$ is with the end to contribute that (55) vanishes adequately.
In accordance with the PSEM, we propose the sum of (55) and (50) (with coefficients (54)) as a solution valid for the whole interval.

$$
y(x)=\frac{\left(a_{2} x^{2}+a_{1} x+a_{0}\right) e^{-2 x}}{\left(b_{9} x^{9}+b_{8} x^{8}+b_{7} x^{7}+b_{6} x^{6}+b_{5} x^{5}+b_{4} x^{4}+b_{3} x^{3}+b_{2} x^{2}+b_{1} x+1\right)}+Y(x)+x-1.7168
$$


TABLE 1: Comparison of the results obtained for this work with other approximations.

\begin{tabular}{|c|c|c|c|c|c|c|c|c|}
\hline$x$ & Exact & $\begin{array}{c}\text { PHPM with } \\
\text { parameters (52) }\end{array}$ & $\begin{array}{c}\text { PHPM with } \\
\text { parameters (54) } \\
\text { and PSEM }\end{array}$ & $\begin{array}{l}\text { LTNHPM } \\
{[33]}\end{array}$ & HPM [1] & $\begin{array}{c}\text { Iteration } \\
\text { perturbation method } \\
(\text { IPM) }[55]\end{array}$ & $\begin{array}{l}\text { Modified } \\
\text { Padé [51] }\end{array}$ & $\begin{array}{c}\text { Weighted residual } \\
\text { method (WRM) } \\
{[56]}\end{array}$ \\
\hline 0.2 & 0.00664 & 0.0751339 & 0.00664 & 0.00664 & 0.008389 & 0.006441 & -- & 0.007170 \\
\hline 0.4 & 0.026600 & 0.0718948 & .0265590 & 0.02656 & 0.033359 & 0.025186 & 0.026600 & 0.027729 \\
\hline 0.6 & 0.05974 & 0.1412990 & 0.059732 & 0.05973 & 0.074368 & 0.055411 & --- & 0.060957 \\
\hline 0.8 & 0.10611 & 0.2072926 & 0.106088 & 0.10611 & 0.130645 & 0.096350 & 0.106100 & 0.107292 \\
\hline 1.0 & 0.16557 & 0.2898499 & 0.165467 & 0.16557 & 0.201275 & 0.147296 & --- & 0.167581 \\
\hline 1.4 & 0.32298 & 0.4951863 & 0.322103 & 0.32298 & 0.381558 & 0.276618 & 0.32290 & 0.331867 \\
\hline 1.6 & 0.42032 & 0.6142525 & 0.418515 & 0.42032 & 0.489145 & 0.353812 & 0.420300 & 0.435454 \\
\hline 1.8 & 0.52952 & 0.7422946 & 0.526322 & 0.52952 & 0.607009 & 0.438643 & -- & 0.552286 \\
\hline 2.2 & 0.78120 & 1.0208130 & 0.774110 & 0.78119 & 0.869751 & 0.629277 & --- & 0.820910 \\
\hline 2.6 & 1.07252 & 1.3233962 & 1.062068 & 1.07250 & 1.162724 & 0.844966 & --- & 1.127496 \\
\hline 2.8 & 1.23099 & 1.4819850 & 1.220049 & 1.23098 & 1.318610 & 0.961224 & 1.231100 & 1.291934 \\
\hline 3.2 & 1.56911 & 1.8111840 & 1.560172 & 1.56909 & 1.645862 & 1.208832 & 1.569300 & 1.637813 \\
\hline 3.6 & 1.92954 & 2.1533835 & 1.924973 & 1.92951 & 1.990023 & 1.474506 & 1.929700 & 2.000738 \\
\hline 3.8 & 2.11305 & 2.3286100 & 2.113736 & 2.11596 & 2.167250 & 1.613421 & --- & 2.186909 \\
\hline 4.0 & 2.30576 & 2.5061893 & 2.305453 & 2.30550 & 2.347369 & 1.756054 & 2.305800 & 2.375521 \\
\hline 4.2 & 2.49806 & 2.6858832 & 2.499383 & 2.49720 & 2.530031 & 1.902174 & --- & 2.566153 \\
\hline 4.4 & 2.69238 & 2.8674868 & 2.694953 & 2.68965 & 2.714924 & 2.051563 & 2.692200 & 2.758453 \\
\hline 4.6 & 2.88826 & 3.0507910 & 2.891728 & 2.88002 & 2.901774 & 2.204020 & 2.887900 & 2.952128 \\
\hline 4.8 & 3.08534 & 3.2356491 & 3.089384 & 3.06157 & 3.090337 & 2.359356 & 3.084800 & 3.146934 \\
\hline 5.0 & 3.28329 & 3.4219049 & 3.287682 & 3.21785 & 3.280397 & 2.517394 & 3.282700 & 3.342676 \\
\hline 5.4 & 3.68094 & 3.7980836 & 3.685553 & --- & 3.664274 & 2.840918 & 3.680500 & 3.736318 \\
\hline 5.8 & 4.07990 & 4.1784119 & 4.084435 & --- & 4.052148 & 3.173388 & 4.079600 & 4.132045 \\
\hline 6.2 & 4.47948 & 4.5621475 & 4.483847 & --- & 4.443052 & 3.513744 & --- & 4.529177 \\
\hline 6.6 & 4.87931 & 4.9486879 & 4.883539 & --- & 4.836243 & 3.861055 & & 4.927252 \\
\hline 7.0 & 5.27926 & 5.3375409 & 5.283378 & --- & 5.231158 & 4.214503 & 5.279200 & 5.325961 \\
\hline 7.4 & 5.67924 & 5.7283035 & 5.683293 & --- & 5.627366 & 4.573365 & 5.679200 & 5.725095 \\
\hline 7.8 & 6.07923 & 6.1206439 & 6.083249 & --- & 6.024545 & 4.937007 & --- & 6.124514 \\
\hline 8.2 . & 6.47923 & 6.5142919 & 6.483225 & --- & 6.422449 & 5.304871 & --- & 6.524125 \\
\hline 8.6 & 6.87923 & 6.9090209 & 6.883213 & --- & 6.820894 & 5.676462 & --- & 6.922386 \\
\hline 8.8 & 7.07923 & 7.1067317 & 7.083209 & --- & 7.020275 & 5.863518 & --- & 7.123767 \\
\hline 10.0 & 8.2792 & 8.2832000 & 8.283201 & --- & 8.218009 & 7.000557 & 8.279200 & 8.323464 \\
\hline
\end{tabular}

The symbol "-_-" means data are not available in the corresponding article.

Next, we obtain a Taylor expansion around $x=0$ for (56), and this result is matched with series (53) (that is, we equate the coefficients of both power series) with the purpose to get an algebraic equation system with the end to determine the coefficients of (55).

As a consequence, we determine the following values:

$$
\begin{aligned}
& a_{0}=-31.1293, \\
& a_{1}=-14.2776, \\
& a_{2}=-30.87080, \\
& b_{1}=1.85423, \\
& b_{2}=1.24959, \\
& b_{3}=0.264101, \\
& b_{4}=-0.032011, \\
& b_{5}=0.0395264, \\
& b_{6}=0.0395466, \\
& b_{7}=-0.0010344, \\
& b_{8}=-0.003506, \\
& b_{9}=0.0024592 .
\end{aligned}
$$

Thus, substituting (57) into (56), we obtain an analytical approximate solution for the BP. From Table 1, we note that the accuracy of (56) with coefficients (54) and (57) is clearly better than the one obtained for the PHPM (comparing the first two columns with the exact solution).

\section{Discussion}

Next, we will present the main results obtained for this work. In particular, we will emphasize the advantages of the proposed PHPM for the case where there exists additional information about the problem to solve.

This article presented a modification of the HPM, the modified HPM (PHPM), with the purpose of obtaining an analytical approximate solution for the relevant BP. Essentially, the procedure follows the standard HPM, but avoiding the integration constants in each iteration. Once one decides what is the last iteration to conclude the iterative procedure, the constants are added in this step, and the final approximate solution contains these parameters. We also mentioned that this scheme could result useful in the cases where, for example, the knowledge of certain mathematics and physical information could aid in knowing the value of some of these parameters (of course, some constants are 
determined from the initial and boundary conditions proposed by the problem). To begin, we were able to express the solution of this problem as the sum of an asymptotic part and other valid for relatively small values of $x$. Along this work, we showed the manner to introduce some conditions with the end to determine the values of some constants. For example, the boundary condition $y^{\prime}(\infty)=1$ implies that asymptotically, the derivative function tends to the constant value one and for the same reason, the dimensionless stream function has to assume, in this same regime, the rather simple form $y(x)=x+c, x \longrightarrow \infty$.

Comparing this asymptotic form with (44), we noted that it is required that the last term of (44) has to be zero; besides, from the same condition, it is clear that $k_{2}=1$. Therefore, the particular form of expressing the PHPM solution easily allows calculating these values. In the same way, we saw that the Blasius asymptotic solution admits a geometry interpretation which allowed knowing the value of $k_{1}$. Therefore, we note the convenience of using the PHPM in order to determinate the asymptotic part of the proposed solution, taking advantage of the mathematics and physical information that concerns with the problem.

On the other hand, the PHPM easily allowed calculating the other unknown parameters $A$ and $B$. This procedure was implemented in order to ensure that (50) satisfied the initial conditions of the problem. In accordance with PHPM algorithm, we substituted (50) into the first condition and the second condition of (27) with the end to obtain an algebraic system for the unknown quantities.

The analysis from Table 1 shows a moderate precision; as a matter of fact, we got the best performance for values next to $x=5$ and higher than this value. This result finds an explanation in the fact that the PHPM solution ensured, from the beginning, the correct behaviour of the asymptotic part (50). Besides, from the same table, we note that the absolute error for values between $x=2.8$ and $x=4.8$ is in the order of two to three hundredths, which represent an acceptable precision. It is expected that, for improving the precision of (50), higher-order iterations be required and/or an initial trial function that looks like (38) be provided but with more adjusting parameters (other trial functions could be tried with the correct asymptotic behaviour of the problem, for example, a sum of two terms like (38), and we will provide fourth adjusting parameters) in order to get a better result.

It is important to note the power and flexibility of the homotopy scheme proposed in this work. However, in future research, we propose exploring alternative ways to construct other homotopy schemes. For example, the homotopy perturbation method with two expanding parameters [28], homotopy perturbation method with three expansions [29], Li-He homotopy perturbation method [30], homotopy perturbation with an auxiliary parameter [31], and He Laplace method [32,33].

7.1. Combination of the PHPM and PSEM. Next, in order to obtain a better approximation, it was proposed to recalculate the parameters $A$ and $B$ mentioned above, but changing the strategy. We conceived to divide the solution of the problem into two parts, the first one valid for the interval $[0,3)$ and the second for the infinite interval $[3, \infty)$.

With respect to the solution in the infinite interval, the procedure consisted in recalculating the parameters (54) with the purpose of obtaining a new analytical approximate solution for (26)-(27) in the mentioned interval.

That is, we repeated the same steps that yield in (50) with parameters (52) but shortening the interval as it was indicated. We expected to improve the above approximation because the solution of the problem at the beginning of the interval is strongly affected, not only from the asymptotic term $x-1.7168$, but for $Y(x)$, while as we consider bigger values of $x$, the asymptotic part becomes the predominant, for which the solution for both regions is indeed different. Therefore, if we ask the PHPM to describe only partially the interval, it is expected to get a better approximation, which, in fact, was what occurred.

The manner to recalculate the values of the $A$ and $B$ of (50) was substituting some points of the solution curve into (50) in order to obtain an algebraic system for the mentioned unknown quantities. The points used for this purpose are $(3,1.39682)$, and $(4,2.30576)$, which were obtained from (53) (see also (28)). After solving the resulting $2 \times 2$ nonlinear system, we got the values (54). It is important to clarify why we did not employ the points correspondent, for instance, to $x=5$ and $x=6$, instead of the points mentioned above which correspond to $x=3$ and $x=4$. By using $x=5$ and $x=6$, the PHPM would have obtained a solution only for the asymptotic part of the problem with a very good expectation to describe it with precision. We emphasize that two points are required for this purpose, which are obtained from the Blasius series. As explained in Section 5, the convergence of this series lies in the interval $[0, \phi]$, where $\phi$ is a number such that $\phi \approx 5.69$.

Thus, to avoid calculating points from the Blasius series too close to its convergence upper end point, we preferred using values of $x$, totally within the convergence interval.

In order to get a good approximation for the interval $[0,3)$, a simple choice would have been to use (28). After all, we have seen that, for $x$ within its convergence interval, even four terms from (28) provide good results (53). In this way, we could provide a piece-wise kind of solution, and employing unit step functions $U(x-a)[3,4]$, we may get a final result by using a compact expression. In a sequence, in theory, it would have been even possible to get a differentiable approximate expression by using differentiable and continuous analytical approximate functions for the step function. However, in general, many times, that mentioned earlier is not possible but only approximated because to match two approximate solutions in the join points is not an easy task.

With the purpose of getting a real continuous and differentiable solution for the whole interval of this problem, we proposed using the PSEM in order to get an accurate solution that includes the interval $[0,3)$.

With this end, the PSEM proposed the rational function (55) and, therefore, the sum of (50) and (55) (with coefficients (54) for (50)) as a solution for the entire interval. Next, 
a Taylor expansion is obtained around $x=0$ for (56), and what results is matched, term by term, with series (53) up to the eleventh power in order to propose an algebraic system of equations to determine the coefficients of (55). This procedure is successful because it not only ensures the correctness of continuity and the differentiability for all the points of the whole interval but also takes advantage of both the accuracy of (53) in the initial part of the interval and the accuracy of the PHPM solution for infinite interval $[3, \infty)$.

Finally, from Table 1, we compared the results obtained for this work with other approximations reported in the literature. In columns two and three appear the approximations discussed for this work.

As commented, in general, the PHPM with parameters (52) had an acceptable precision. Our approximation showed a bad approximation in the beginning of the interval, and after a gradual improvement, we note that, from $x=4$ onwards, the absolute error committed is less than 0.2 ; in particular, for relatively large values of $x$, better results are obtained. Compared with the PHPM (54) and PSEM, the PHPM (52) obtained a lower performance, and the reasons were, in part, the subject of Section 6. Nevertheless, it is important to note that the performance of the PHPM is essentially the same for big values of $x$.

On the other hand, the LTNHPM [57] showed a better performance than the PHPM (52), but it only provides results from $x=0$ to $x=5$, whereby the proposed solution from the PHPM offered a better performance in general. In this work [57], a series with good results was found, but in general, the power series requires many terms in order to get an accurate approximate solution when it is applied to differential equations. As a matter of fact, the series that gave rise to values in Table 1 required 13 terms, and as we consider bigger values of $x$, more and more terms of the series are needed, which is not convenient for practical applications. From the results of Table 1, it is clear that [1] presents a better precision than the PHPM (52) for $x$ values comprised between $x=0$ and $x=7$, while from $x=7$ onwards, the PHPM (52) is better. It ratifies the correctness of the implemented strategy for the PHPM with the purpose of calculating the asymptotic part of the BP. Another point to consider is unlike the proposed method, the method in [1] calculated its unknown coefficients by using an optimization method, the least square method, which represents an advantage for the method in [1].

With respect to the comparison between the PHPM (52) and IPM [55], Table 1 shows that the PHPM provides a better accuracy, except for values comprised from $x=0$ to $x=2.6$, which is very early in the interval. In general, the IPM offered the lower performance of the compared methods. On the contrary, Ahmad et al. [51] showed the best precision, which is deduced from Table 1 . Nevertheless, Ahmad et al. [51] employed a Padé [4/3] approximant of the derivative of (53) as the starting point and added corrective terms both in the numerator and denominator of the mentioned Padé to propose a new expression which yielded good precision. This proposal even successful for the BP is not a general procedure for finding solutions to nonlinear differential equations. Finally, the comparison of the

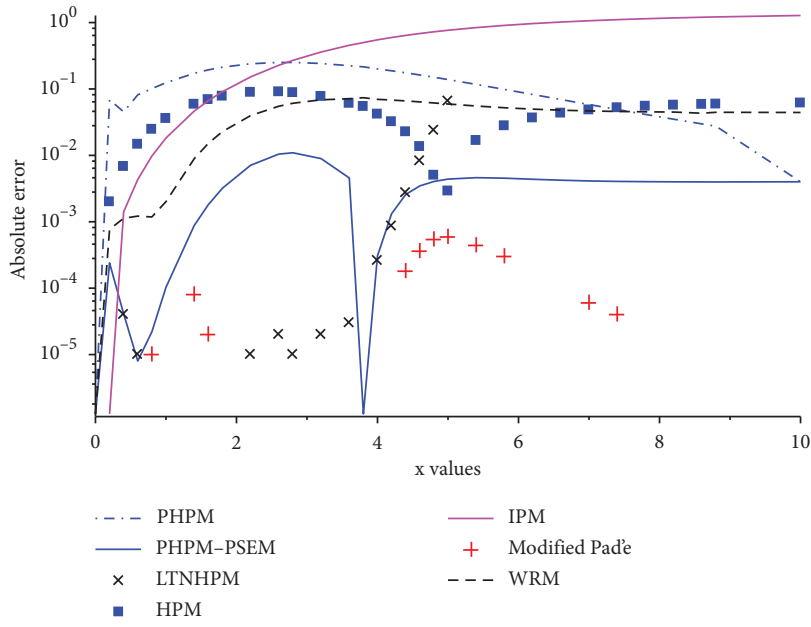

FIgURe 1: Absolute errors committed by using the PHPM (50) with coefficients (52), PHPM-PSEM (56) with coefficients ((54) and (57)), and other approximations from the literature for the proposed problem.

proposed solution PHPM (52) with [56] shows that the proposal from the WRM is better for interval $[0,6.8]$ although the PHPM was competitive in that interval except at the early small values close to zero. Again, the PHPM (52) was better for the asymptotic part of the solution.

As explained, in order to improve the PHPM (52) approximate solution, we introduced the combination of the PHPM and PSEM. From Table 1, we noted that the obtained result was remarkably better. The combination of the PHPM (54) and PSEM is more precise than the PHPM (52), and it is better than the LTNHPM [57] although in interval $[0,5]$, the performance of both was very similar. In the same way, the proposed solution was better than that in [1] although this employed an optimization procedure to calculate its coefficients. Likewise, we note that the PHPM (54) and PSEM were clearly better than the IPM and also overcome the WRM despite that this last is a method widely recognized. In this case, both methods achieved a good performance. Finally, as already said, the method in [51] obtained the best accuracy, but the difference of accuracy between the solutions obtained by the PHPM (54) and PSEM [51] was, most of the times, of only thousandths. An important point is that unlike the one proposed by this work, Ahmad et al. [51] just provided a particular procedure valid in principle for the Blasius problem. Finally, even we could present other HPM approximations for the Blasius equation [21], but this article only presented results for the short interval $[0,4]$. Figure 1 shows the absolute error committed for the method solutions exposed earlier.

In brief, the results obtained for the PSEM combined with the PHPM showed that it is possible to couple two methods, which, in principle, are very different, but combining them can provide a total solution with a wider domain; as a matter of fact, the PHPM delivered the asymptotic part of the problem and the PSEM focused on solving the part closer to $x=0$, in such a way that the coupling of both methods yielded a new function defined for the whole 
interval with good precision, as discussed, in relation with Table 1. The matter of improving the convergence of the PHPM and PHPM and PSEM combination could be considered in a future theme of investigation. As a matter of fact, methodologies such as those considered in $[24,30]$ could be useful with this purpose.

\section{Conclusions}

This work introduced a modification of the standard HPM, the PHPM, which is particularly useful when it is possible to dispose additional information, both mathematical and physical, for a proposed nonlinear problem. Unlike the HPM which establishes that the values of higher-order approximations have to be zero if they are evaluated for the initial condition, the PHPM proposed that the successive integrations process of the coupled system (7)-(10) is performed without evaluating the initial conditions mentioned by the HPM, considering zero the constants of integration in each iteration, except for the last one, which will keep all its integration constants that are originated by the process. The whole solution (11) will possess some integration constants to evaluate even other parameters that could rise from the freedom of the homotopy technique, and the PHPM evaluates these quantities from the initial conditions applied to (11) and the knowledge of other mathematical and physical properties of the nonlinear problem. The proposed PHPM expresses in a natural way the solution as a sum of an asymptotic part and other part that describes the solution for $x$ values closer to the origin. From the evidence presented for this article, it is expected that

PHPM contributes to change the idea that an effective method has to be necessarily long and cumbersome as occurs with the HAM and Adomian decomposition method, among others

the proposal of PHPM is that of a method that is both precise and easy to implement

the proposed method allows incorporating the knowledge of the mathematical and physical properties that emanate from the problem to solve and not only the initial conditions

On the other hand, as occurred in this work, sometimes, it is expected that, for improving the precision of the proposed solutions for the PHPM it is required both to obtain higher order iterations and to introduce an initial trial function provided with several adjusting parameters, which could lead to a cumbersome procedure. To avoid that inconvenience, this work improved the results obtained by the PHPM by using a combination of the PHPM and PSEM which showed to have high potential for practical problems. The success of the combination is that the PSEM took advantage of the good performance of a known series solution for the initial part of the interval and the PHPM provided the asymptotic part of the solution with good accuracy. In summary, the PHPM and the union of the PSEM with PHPM demonstrated potential for future applications.

\section{Nomenclature}

BP: $\quad$ Blasius problem

HPM: $\quad$ Homotopy perturbation method

PHPM: $\quad$ Powered homotopy perturbation method

PSEM: $\quad$ Power series extender method

PM: $\quad$ Perturbation method

BODE: Blasius ordinary differential equation

LTNHPM: Laplace transform coupled with the new homotopy perturbation method

IPM: Iteration perturbation method

WRM: Weighted residual method.

\section{Data Availability}

The data used to support the findings of this study are included within the article.

\section{Conflicts of Interest}

The authors declare no conflicts of interest regarding the publication of this paper.

\section{Acknowledgments}

The authors would like to thank Rogelio Alejando CallejasMolina and Roberto Ruiz Gomez for their contribution to this project.

\section{References}

[1] U. Filobello-, H. Vazquez-Le, R. Castaneda- et al., "An approximate solution of blasius equation by using hpm method," Asian Journal of Mathematics \& Statistics, vol. 5, no. 2, pp. 50-59, 2012.

[2] L. D. Landau and E. M. Lifshitz, "Theoretical physics," Fluid Mechanics, vol. 6, 1987.

[3] U. Filobello-Nino, H. Vazquez-Leal, Y. Khan et al., "A handy exact solution for flow due to a stretching boundary with partial slip," Revista mexicana de física E, vol. 59, no. 51-55, 2013.

[4] L. M. B. Assas, "Approximate solutions for the generalized KdV-Burgers' equation by He's variational iteration method," Physica Scripta, vol. 76, no. 2, pp. 161-164, 2007.

[5] M. Kazemnia, S. A. Zahedi, M. Vaezi, and N. Tolou, "Assessment of modified variational iteration method in bvps high-order differential equations," Journal of Applied Sciences, vol. 8 , no. 22, pp. 4192-4197, 2008.

[6] R. Noorzad, A. T. Poor, and M. Omidvar, "Variational iteration method and homotopy-perturbation method for solving burgers equation in fluid dynamics," Journal of Applied Sciences, vol. 8, no. 2, pp. 369-373, 2008.

[7] D. J. Evans and K. R. Raslan, "The tanh function method for solving some important non-linear partial differential equations," International Journal of Computer Mathematics, vol. 82, no. 7, pp. 897-905, 2005.

[8] F. Xu, "A generalized soliton solution of the konopelchenkodubrovsky equation using He's exp-function method," Zeitschrift für Naturforschung A, vol. 62, no. 12, pp. 685-688, 2007.

[9] J. Mahmoudi, N. Tolou, I. Khatami, A. Barari, and D. D. Ganji, "Explicit solution of nonlinear zk-bbm wave equation using 
exp-function method," Journal of Applied Sciences, vol. 8, no. 2, pp. 358-363, 2008.

[10] G. Adomian, "A review of the decomposition method in applied mathematics," Journal of Mathematical Analysis and Applications, vol. 135, no. 2, pp. 501-544, 1988.

[11] E. Babolian and J. Biazar, "On the order of convergence of adomian method," Applied Mathematics and Computation, vol. 130, no. 2, pp. 383-387, 2002.

[12] K. Ali and M. Abadyan, "Efficiency of modified adomian decomposition for simulating the instability of nano-electromechanical switches: comparison with the conventional decomposition method," Trends in Applied Sciences Research, vol. 7 , no. 1, p. 57, 2012.

[13] K. Ali and M. Abadyan, "Evaluating the ability of modified adomian decomposition method to simulate the instability of freestanding carbon nanotube: comparison with conventional decomposition method," Journal of Applied Sciences, vol. 11, no. 19, pp. 3421-3428, 2011.

[14] S. K. Vanani, S. Heidari, and M. Avaji, "A low-cost numerical algorithm for the solution of nonlinear delay boundary integral equations," Journal of Applied Sciences, vol. 11, no. 20, pp. 3504-3509, 2011.

[15] S. Hossien Chowdhury, "A comparison between the modified homotopy perturbation method and adomian decomposition method for solving nonlinear heat transfer equations," Journal of Applied Sciences, vol. 11, no. 7, pp. 1416-1420, 2011.

[16] L.-N. Zhang and L. Xu, "Determination of the limit cycle by he's parameter-expansion for oscillators in a $\mathrm{u} 3 /(1+\mathrm{u} 2)$ potential," Zeitschrift für Naturforschung A, vol. 62, no. 7-8, pp. 396-398, 2007.

[17] H. Aminikhah and M. Hemmatnezhad, "A novel effective approach for solving nonlinear heat transfer equations," Heat Transfer-Asian Research, vol. 41, no. 6, pp. 459-467, 2012.

[18] J.-H. He, "Homotopy perturbation method for solving boundary value problems," Physics Letters A, vol. 350, no. 1, pp. 87-88, 2006.

[19] J. -H. He, "Recent development of the homotopy perturbation method," Topological Methods in Nonlinear Analysis, vol. 31, no. 2, pp. 205-209, 2008.

[20] J. -H. He, "A coupling method of a homotopy technique and a perturbation technique for non-linear problems," International Journal of Non-linear Mechanics, vol. 35, no. 1, pp. 37-43, 2000.

[21] D. D. Ganji, H. Babazadeh, F. Noori, M. M. Pirouz, and M. Janipour, "An application of homotopy perturbation method for non-linear blasius equation to boundary layer flow over a flat plate," International Journal of Nonlinear Science, vol. 7, no. 4, pp. 399-404, 2009.

[22] U. Filobello-Nino, H. Vazquez-Leal, A. Sarmiento-Reyes et al., "Laplace transform-homotopy perturbation method with arbitrary initial approximation and residual error cancelation," Applied Mathematical Modelling, vol. 41, pp. 180194, 2017.

[23] U. Filobello-Nino, H. Vazquez-Leal, M. M. Rashidi et al., "Laplace transform homotopy perturbation method for the approximation of variational problems," SpringerPlus, vol. 5, no. 1, pp. 1-33, 2016.

[24] X.-X. Li and C.-H. He, "Homotopy perturbation method coupled with the enhanced perturbation method," Journal of Low Frequency Noise, Vibration and Active Control, vol. 38, no. 3-4, pp. 1399-1403, 2019.

[25] J.-H. He and Y. O. El-Dib, "Homotopy perturbation method for fangzhu oscillator," Journal of Mathematical Chemistry, vol. 58, no. 10, pp. 2245-2253, 2020.
[26] Y. O. El-Dib, G. M. Moatimid, and N. S. Elgazery, "Stability analysis of a damped nonlinear wave equation," Journal of Applied and Computational Mechanics, vol. 6, pp. 1394-1403, 2020.

[27] N. Anjum and Q. Tul Ain, "Application of he's fractional derivative and fractional complex transform for time fractional camassa-holm equation," Thermal Science, vol. 24, no. 5, pp. 3023-3030, 2020.

[28] J. H. He, "Homotopy perturbation method with two expanding parameters," Indian Journal of Physics, vol. 88, no. 2, pp. 193-196, 2014.

[29] J.-H. He and Y. O. El-Dib, "Homotopy perturbation method with three expansions," Journal of Mathematical Chemistry, vol. 59, no. 4, pp. 1139-1150, 2021.

[30] N. Anjum, J.-H. He, Q. Tul Ain, and D. Tian, "Li-he's modified homotopy perturbation method for doubly-clamped electrically actuated microbeams-based microelectromechanical system," Facta Universitatis, Series: Mechanical Engineering, vol. 21, 2021.

[31] D.-N. Yu, J.-H. He, and A. G. Garcia, "Homotopy perturbation method with an auxiliary parameter for nonlinear oscillators," Journal of Low Frequency Noise, Vibration and Active Control, vol. 38, no. 3-4, pp. 1540-1554, 2019.

[32] J.-H. He, G. M. Moatimid, and D. R. Mostapha, "Nonlinear instability of two streaming-superposed magnetic ReinerRivlin Fluids by He-Laplace method," Journal of Electroanalytical Chemistry, vol. 895, Article ID 115388, 2021.

[33] N. Anjum, C.-H. He, and J. -H. He, Two-scale Fractal Theory for the Population Dynamics, Fractals, Singapore, 2021.

[34] U. Filobello-Nino, H. Vazquez-Leal, A. Sarmiento-Reyes et al., "The study of heat transfer phenomena using pm for approximate solution with dirichlet and mixed boundary conditions," Applied and Computational Mathematics, vol. 2, no. 6, pp. 143-148, 2013.

[35] U. Filobello-Nino, H. Vazquez-Leal, Y. Khan et al., "Using perturbation methods and Laplace-Padé approximation to solve nonlinear problems," Miskolc Mathematical Notes, vol. 14, no. 1, pp. 89-101, 2013.

[36] U. Filobello-Nino, H. Vazquez-Leal, B. Benhammouda et al., "A handy approximation for a mediated bioelectrocatalysis process, related to michaelis-menten equation," Springer Plus, vol. 3, no. 1, pp. 1-6, 2014.

[37] H. Vazquez-Leal, M. Sandoval-Hernandez, R. CastanedaSheissa, U. Filobello-Nino, and A. Sarmiento-Reyes, "Modified taylor solution of equation of oxygen diffusion in a spherical cell with michaelis-menten uptake kinetics," International Journal of Applied Mathematical Research, vol. 4, no. 2, p. 253, 2015.

[38] U. Filobello-Nino, H. Vazquez-Leal, A. Perez-Sesma et al., "On a practical methodology for solving BVP problems by using a modified version of picard method," Applied Mathematics \& Information Sciences, vol. 10, no. 4, pp. 1355-1367, 2016.

[39] H. Vazquez-Leal and A. Sarmiento-Reyes, "Power series extender method for the solution of nonlinear differential equations," Mathematical Problems in Engineering, vol. 2015, Article ID 717404, 7 pages, 2015.

[40] H. Vazquez-Leal, J. L. Garcia-Gervacio, U. A. Filobello-Nino, M. A. Sandoval-Hernandez, and A. L. Herrera-May, "Psem approximations for both branches of lambert $\mathrm{w}$ function with applications," Discrete Dynamics in Nature and Society, vol. 2019, Article ID 8267951, 9 pages, 2019.

[41] U. Filobello-Nino, H. Vazquez-Leal, A. L. Herrera-May et al., "A handy, accurate, invertible and integrable expression for 
dawson's function," Acta Universitaria, vol. 29, Article ID e2124, 2020.

[42] M. A. Sandoval-Hernandez, H. Vazquez-Leal, U. FilobelloNino, and L. Hernandez-Martinez, "New handy and accurate approximation for the Gaussian integrals with applications to science and engineering," Open Mathematics, vol. 17, no. 1, pp. 1774-1793, 2019.

[43] S. Liao, "Homotopy analysis method: a new analytic method for nonlinear problems," Applied Mathematics and Mechanics, vol. 19, no. 10, pp. 957-962, 1998.

[44] J. -H. He and X. -H. Wu, "Variational iteration method: new development and applications," Computers \& Mathematics with Applications, vol. 54, no. 7, pp. 881-894, 2007.

[45] J. M. W. Munganga, J. N. Mwambakana, R. Maritz, T. A. Batubenge, and G. M. Moremedi, "Introduction of the differential transform method to solve differential equations at undergraduate level," International Journal of Mathematical Education in Science and Technology, vol. 45, no. 5, pp. 781-794, 2014.

[46] M. M. Peiravi, J. Alinejad, D. Ganji, and S. Maddah, "Numerical study of fins arrangement and nanofluids effects on three-dimensional natural convection in the cubical enclosure," Transport Phenomena in Nano and Micro Scales, vol. 7, no. 2, pp. 97-112, 2019.

[47] M. M. Peiravi and J. Alinejad, "Hybrid conduction, convection and radiation heat transfer simulation in a channel with rectangular cylinder," Journal of Thermal Analysis and Calorimetry, vol. 140, no. 6, pp. 2733-2747, 2020.

[48] J. Alinejad and M. M. Peiravi, "Numerical analysis of secondary droplets characteristics due to drop impacting on $3 \mathrm{~d}$ cylinders considering dynamic contact angle," Meccanica, vol. 55, no. 10, pp. 1975-2002, 2020.

[49] M. M. Peiravi, J. Alinejad, D. D. Ganji, and S. Maddah., "3d optimization of baffle arrangement in a multi-phase nanofluid natural convection based on numerical simulation," International Journal of Numerical Methods for Heat \& Fluid Flow, vol. 30, no. 5, pp. 2583-2605, 2020.

[50] D. G. Zill, A First Course in Differential Equations with Modeling Applications, Cengage Learning, Boston, MA, USA, 2012.

[51] F. Ahmad, W. H. Al-Barakati, and A. - Barakati, "An approximate analytic solution of the blasius problem," Communications in Nonlinear Science and Numerical Simulation, vol. 14, no. 4, pp. 1021-1024, 2009.

[52] B. Heinrich, "Grenzschichten in flüssigkeiten mit kleiner reibung," Zeitschrift für angewandte Mathematik und Physik, vol. 56, no. 1-37, 1908.

[53] L. Howarth and L. Bairstow, "On the solution of the laminar boundary layer equations," Proceedings of the Royal Society A: Mathematical, Physical and Engineering Sciences, vol. 164, no. 919 , pp. 547-579, 1938.

[54] A. Asaithambi, "Solution of the Falkner-Skan equation by recursive evaluation of Taylor coefficients," Journal of Computational and Applied Mathematics, vol. 176, no. 1, pp. 203-214, 2005.

[55] H. E. Ji-Huan, "Some asymptotic methods for strongly nonlinear equations," International Journal of Modern Physics B, vol. 20, no. 10, pp. 1141-1199, 2006.

[56] U. C. Karabulut and A. Kiliç, "Blasius denkleminin çözümü için çeşitli teknikler,” Balıkesir Üniversitesi Fen Bilimleri Enstitüsü Dergisi, vol. 20, pp. 129-142, 2018.

[57] H. Aminikhah, "Analytical approximation to the solution of nonlinear blasius' viscous flow equation by ltnhpm," ISRN Mathematical Analysis, vol. 2012, Article ID 957473, 9 pages, 2012. 